\title{
The magnetic canopy above light bridges
}

\author{
J. Jurčák ${ }^{1}$, V. Martínez Pillet ${ }^{2}$, and M. Sobotka ${ }^{1}$ \\ 1 Astronomical Institute, Academy of Sciences of the Czech Republic, 25165 Ondřejov, Czech Republic \\ e-mail: jurcak@asu.cas.cz \\ 2 Instituto de Astrofísica de Canarias, 38200 La Laguna, Tenerife, Spain
}

Received 4 November 2005 / Accepted 26 January 2006

\begin{abstract}
An analysis of high-resolution Stokes observations of two light bridges in active region NOAA 8990 is presented. The observations were recorded with the La Palma Stokes Polarimeter attached to the Swedish Vacuum Solar Telescope. The stratification over the solar atmosphere of different physical parameters is retrieved from these data using the Stokes inversion based on response functions (SIR). Our results confirm previous observations of features such as the decrease in magnetic field strength and the increase in inclination in the light bridges. We also confirm a temperature increase in these structures with respect to the surrounding umbrae. The maps of the magnetic field strength and of the orientation of the magnetic field vector indicate the presence of a canopy structure above the light bridges. We derive the vertical component of electric current density $\left(J_{z}\right)$ from the configuration of the magnetic field. The increased temperature found in the upper layers is studied in the context of the proposed canopy topology and could also explain the recently observed chromospheric heating processes found above light bridges.
\end{abstract}

Key words. sunspots - Sun: magnetic fields

\section{Introduction}

Light bridges (hereafter LBs) are bright structures in sunspots that have been known for a long time. They separate umbral cores or penetrate deep into them. In spite of how well-observed LBs are, only the basic characteristics of these structures are known.

The formation of LBs either indicates the imminent breakup of the sunspot in the decay phase or it may occur during the assembly process in complex active regions (Bray \& Loughhead 1964; García de La Rosa 1987). The LBs have various shapes, intensities, and sizes (their width varies from less than $1^{\prime \prime}$ to several seconds of arc). Previous studies have tried to categorise them according to various morphological classifications based, for example, on "geometrical" arrangement, brightness, or the magnetic polarity of surrounding umbral cores. By geometrical arrangement we mean the classification made by Sobotka et al. (1994), where LB separating umbral cores is called "strong" and a penetration of brighter material into the sunspot umbra is called a "faint" LB. The classification based on intensity has basically three possibilities - "photospheric", "penumbral", and "umbral". The photospheric granular-like material fills the broadest LBs, while the narrowest ones resemble chains of umbral dots (Bumba \& Suda 1983; Sobotka et al. 1993). The internal structure depends on the inclination of the local magnetic field and can be granular, filamentary, or a combination of both. The polarity arrangement has only two possibilities, separation of the umbral cores with the same or opposite magnetic polarity (Bumba \& Hejna 1980; Zirin \& Wang 1990; Sobotka et al. 1994). The classification of LBs is not unambiguous because they span more than one morphological category.

Previous papers report the common characteristics of the LB magnetic field structure. The magnetic field is generally weaker and more inclined with respect to the local vertical
(Beckers \& Schröter 1969; Lites et al. 1991; Rüedi et al. 1995; Leka 1997).

Our paper concentrates here on magnetic field topology, and we intend to study the velocity structure in future work; however, we briefly report on the present knowledge about this topic. There are no systematic findings concerning the velocities in LBs. Beckers \& Schröter (1969) found blueshifts with respect to the umbral velocities, Rüedi et al. (1995) found redshifts, and Leka (1997) found either blueshifts or redshifts in 11 different LBs studied in her paper. Convective elements similar to granulation with upflows in bright granules and downflows in dark lanes are observed in granular LBs (Rimmele 1997). In comparison with photospheric granulation, cell sizes and velocities in LBs are smaller and lifetimes longer, which may be a consequence of the remaining weak magnetic field. Observations of evolution and horizontal motions of bright granules in LBs also indicate the existence of convective motions (Hirzberger et al. 2002). Recently, rapidly changing chromospheric brightenings above LBs have been found (Berger \& Berdyugina 2003). From the above-mentioned facts it can be concluded that LBs are deepformed structures created by field-free (or weak-field) plasma intruding into an otherwise stable magnetic sunspot.

In this paper we present an analysis of two LBs observed with the La Palma Stokes Polarimeter (LPSP) attached to the Swedish Vacuum Solar Telescope (SVST). Both LBs studied in this paper separate umbral cores of equal polarities. The observation is described in Sect. 2, including the description of the inversion code. In Sect. 3, some interesting profiles are studied with respect to the resulting atmospheric model, and the resulting maps of plasma parameters are also shown there. In Sect. 4, we present the derivation of the $z$ component of current density and the corresponding Joule heating. We also suggest the canopy structure from the resulting maps of plasma parameters. 


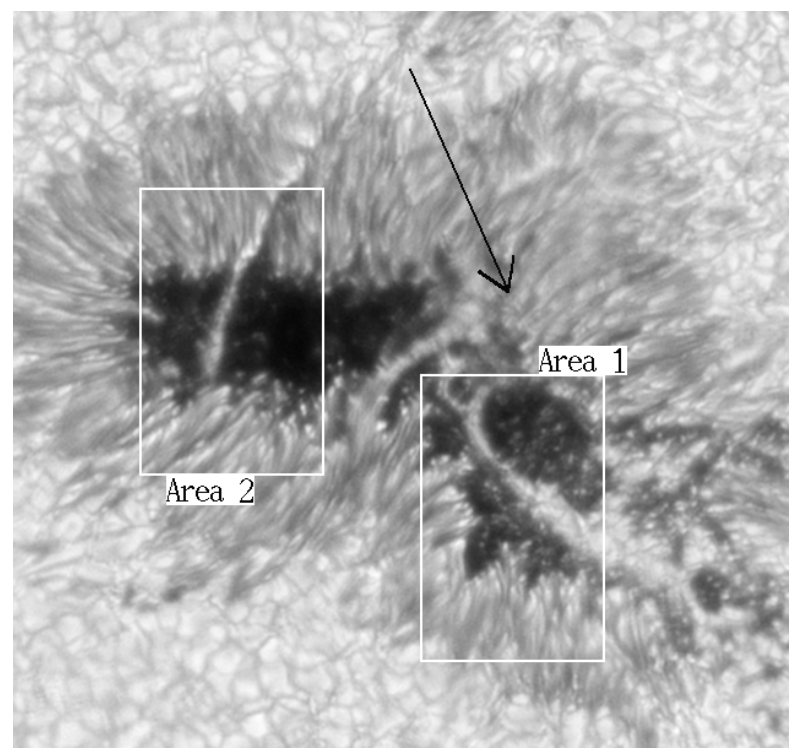

Fig. 1. White-light image of the leading sunspot in NOAA 8990 with the marked areas under study. The arrow points to the north direction.

\section{Observations and data processing}

\subsection{Observations}

On May 13, 2000 two LBs in an irregular sunspot in the active region NOAA 8990 were observed with LPSP (Martínez Pillet et al. 1999) attached to the spectrograph of the $0.5 \mathrm{~m} \mathrm{SVST}$, La Palma (Scharmer et al. 1985). The spot was located near the disc centre, at heliocentric position $14^{\circ} \mathrm{N}$ and $17^{\circ} \mathrm{W}$ (heliocentric angle $\mu=0.907)$. The two maps analysed were taken at 9:03 and 9:13 UT.

White-light images at $\lambda=525 \mathrm{~nm}$ were taken simultaneously with the spectral scans. One of them is shown in Fig. 1. Two areas $(1,2)$ with two distinct LBs (LB1, LB2) were scanned in the magnetically sensitive lines Fe I $630.15 \mathrm{~nm}$ (Landé factor $g=1.67)$ and Fe I $630.25 \mathrm{~nm}(g=2.5)$.

The pixel size of the imaging camera was 0.083 , the resulting resolution including the seeing was around 0.3 , and the exposure time $11 \mathrm{~ms}$. The pixel size of the spectropolarimetric camera was $0{ }^{\prime} 078$. The width of the spectrograph slit was equivalent to 0.32 and the scanning step was 0.24 . The resulting spatial resolution, including the effect of seeing, was about 0.7 for the spectropolarimetric observations. The exposure time for one position of the spectrograph slit was $3 \mathrm{~s}$, in which all Stokes profiles $(I, Q, U$, and $V)$ were acquired.

The telescope instrumental polarisation is fully accounted for, as explained by Martínez Pillet et al. (2001). Two ferroelectric liquid crystals produced four modulation states (linear combinations of the Stokes parameters) with polarisation efficiencies (see Martínez Pillet et al. 2001) of $\epsilon_{Q}=0.38, \epsilon_{U}=0.41$, and $\epsilon_{V}=0.57$. For each slit position, 20 accumulations with $33.3 \mathrm{~ms}$ exposure time each were taken. The signal-to-noise ratio of the reduced data is 600 for the linear polarisation profiles and 800 for the circular polarisation signals. The resulting field of view (approximately $15^{\prime \prime} \times 20^{\prime \prime}$ ) was composed of 80 slit positions in steps of 0.24 . The steps were made by the tip-tilt mirror that was always in closed loop with the sunspot-tracker camera during these two scans. Other details about the observation can be found in Socas-Navarro et al. (2004).

Flatfields were created separately for each polarisation modulation state because they display different interference patterns.
The flatfield data were used to derive the line curvature produced by the spectrograph and passed to the real data for its correction. After darkfield subtraction, flatfield division, and line curvature correction, the data were demodulated with a matrix inferred from the polarisation calibration optics used by the polarimeter. Finally, the Mueller matrix was corrected with the convention of positive $Q$ orientated parallel to the solar rotation axis. Data reduction ended with an ad-hoc correction for crosstalk with an intensity that forces the continuum portions of Stokes $Q, U$, and $V$ to zero.

\subsection{Inversion method}

We used the inversion code SIR (Stokes Inversion based on Response functions) developed at the Instituto de Astrofísica de Canarias (Ruiz Cobo \& del Toro Iniesta 1992). It is a onedimensional code working under the assumption of local thermodynamic equilibrium (LTE). See survey studies by Ruiz Cobo (1998) and Westendorp Plaza et al. (1998, 2001).

The inversion code SIR computes the synthetic Stokes profiles by solving the radiative transfer equation for polarised light in a one-component model atmosphere. A non-linear, leastsquare Marquardt's algorithm (Press et al. 1986) is used to modify the parameters of an initial model until the following merit function is minimised:

$\chi^{2} \propto \sum_{k=1}^{4} \sum_{i=1}^{n}\left[I_{\mathrm{obs}}\left(\lambda_{i}\right)-I_{\mathrm{syn}}\left(\lambda_{i}\right)\right]^{2}$,

where $I_{\mathrm{obs}}\left(\lambda_{i}\right)$ is the observed intensity for the wavelength $\lambda_{i}$, $I_{\text {syn }}\left(\lambda_{i}\right)$ is the synthetic intensity, $k$ samples the 4-component vector of Stokes profiles, and $n$ is the total number of wavelength points. Bellot Rubio (1999) has shown that the error of the plasma parameter $x$ at the optical depth $\tau$ is

$$
\sigma_{x(\tau)}^{2} \propto \frac{\chi^{2}}{\sum_{i=1}^{n} R_{x}^{2}\left(\lambda_{i}, \tau\right)}
$$

where $\chi^{2}$ is the merit function and $R_{x}$ the response function (RF) of the plasma parameter $x$, which describes the sensitivity of the emergent Stokes spectrum to the change $\delta x(\tau)$. The uncertainties are proportional to the merit function and to the inverse of the RF; therefore, poor fits have larger errors, and parameters that have little influence on the emergent intensity show the largest uncertainties.

The SIR code uses a predefined stray-light profile to fit the observed profiles better. We used the stray-light profile coming from the quiet sun (i.e. no stray light in $Q, U$, and $V$ ), but we also made some tests with the stray-light profiles mixed from the quiet-sun profiles and the umbral ones. The details are presented in the next section.

The inversion code returns the stratification of the temperature $(T)$, the magnetic field strength $(B)$, inclination $\left(\gamma_{\mathrm{LOS}}\right)$, and azimuth $\left(\psi_{\text {LOS }}\right)$, along with the line-of-sight velocity, the macroand micro-turbulent velocities, electron pressure, gas pressure, density, and the fraction of the stray light. The transverse-field component direction is unsigned, so that there is a $180^{\circ}$ ambiguity in azimuth values. We used the interactive AZAM code (IDL program written by Seagraves; see Lites et al. 1995, Appendix A) to solve the ambiguity problem. The corrected values of azimuth along with inclination of the magnetic field coming from the SIR code are evaluated with respect to the line of sight. We transformed these variables to the local reference frame; hereafter, the inclination $(\gamma)$ is the angle between 

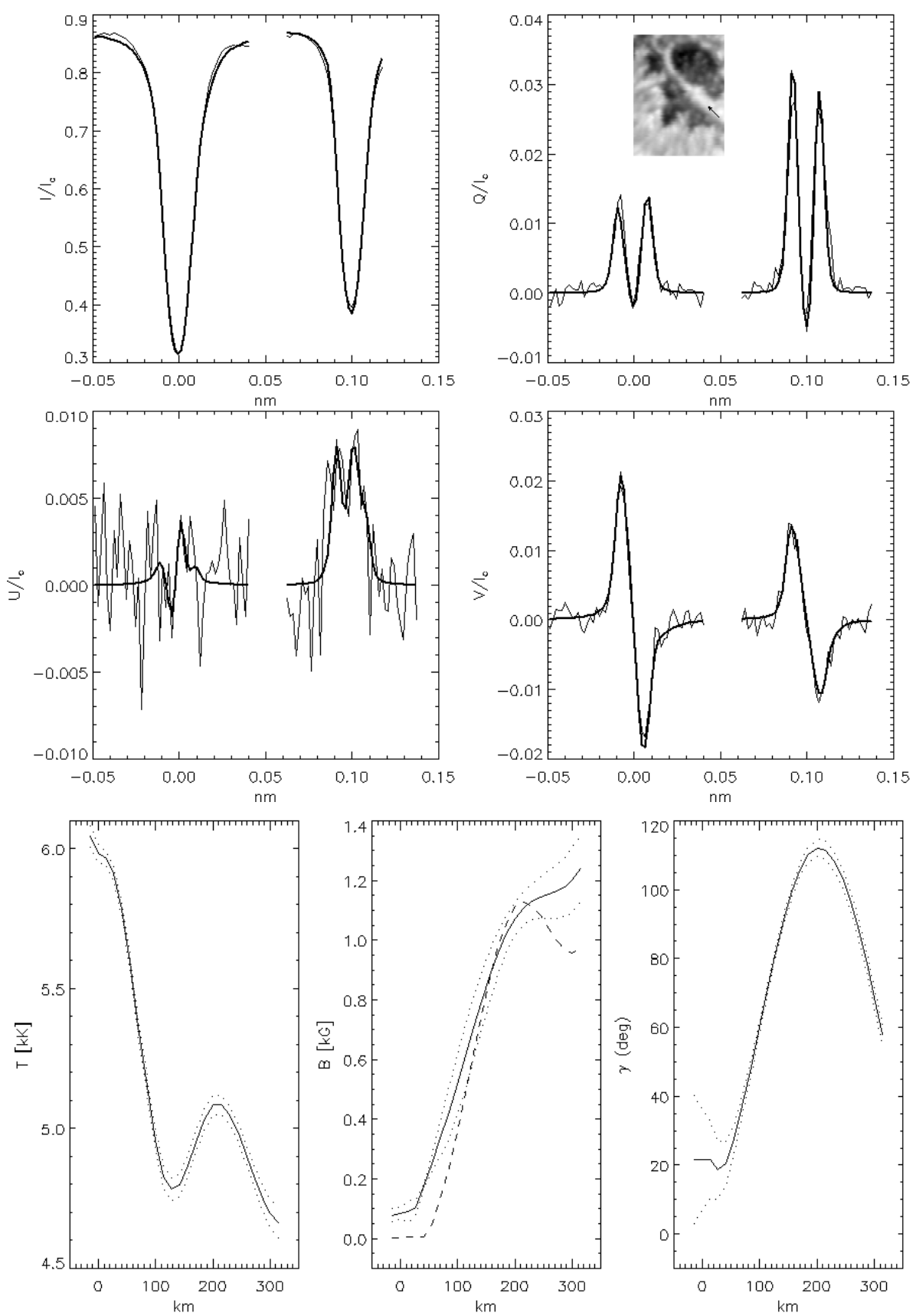

Fig. 2. Upper part: $I, Q, U$, and $V$ profiles of Fe I $6301.5 \AA$ (left) and Fe I $6302.5 \AA$ (right). The observed profile (thin line) is compared with the profile computed by SIR (thick line). The arrow in the white-light image points to the location from which the profiles were obtained, a broad part of LB1. Lower part: the stratification of $T,|B|$, and $\gamma$ (solid line) and the range of errors (dotted line). The dashed line in the $B$ stratification corresponds to a different stray-light profile.

the magnetic field vector, and the local normal line and the azimuth $(\psi)$ is reckoned from the north and increases counterclockwise.

\section{Results of inversions}

\subsection{Stokes profiles and corresponding plasma parameters}

In this section, we describe the most evident differences between the typical Stokes profiles observed in the broad part of LB in area 1 (LB1, Fig. 2) and the typical profiles coming from the narrow parts of LBs (LB2 is shown in Fig. 3). Such differences have a clear impact on the resulting stratification of various plasma parameters. The stratifications of temperature, magnetic field strength, and inclination are plotted with solid lines in the lower part of these figures, together with the 1- $\sigma$ error limits of these parameters (dotted lines).

In the narrow parts of LBs (Fig. 3), we see that the linear polarisation profiles $(Q, U)$, which are highly dependent on the transverse magnetic field component, have smaller amplitudes than the $V$ profile, whose intensity and shape are more directly linked with the longitudinal magnetic field component. This means that the inclination of magnetic field vector has to be smaller than that in the broad part of LB1 (Fig. 2), where the amplitude of the $Q$ profile is comparable to, or even larger than, that of the $V$ profile. The stratification of inclination retrieved by 

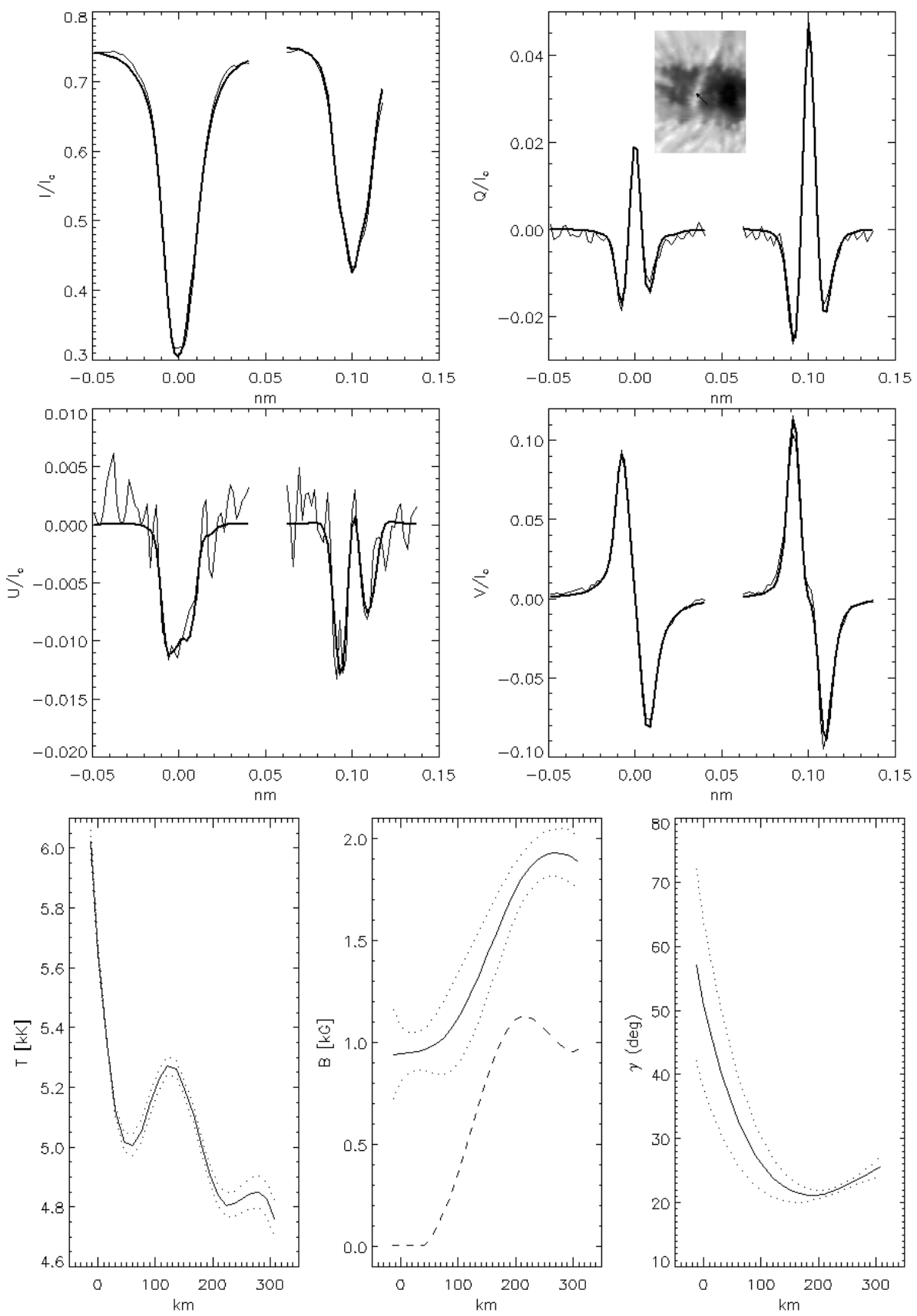

Fig. 3. Same as in Fig. 2 but these profiles were obtained in narrow LB in area 2 (LB2).

SIR confirms this expectation at almost all heights. Inclinations larger than 40 degrees are found at nearly all heights, reaching values of $80^{\circ}-100^{\circ}$.

It is well known that the Fe I line at $6302.5 \AA$ is weaker than at $6301.5 \AA$, and thus, the formation region of the former line lies lower in the photosphere. It is also known that this line is more sensitive to the magnetic field $(g=2.5)$ than the Fe I line at $6301.5 \AA(g=1.67)$. Despite this fact, we see that in the case of the broad part of LB1 (Fig. 2) the amplitude of the $V$ profile of $6301.5 \AA$ is larger than that of $6302.5 \AA$. This could be explained only by a weaker magnetic field strength in the lowest part of the line-formation region, and this is exactly what we see in the plot of the magnetic field stratification in Fig. 2. There $B$ is approximately 100 Gauss in the lowest layers and 1200 Gauss at the height of $200 \mathrm{~km}$. A similar change of $B$ with height (900 Gauss) also occurs in the case of the narrow part of LB2 (Fig. 3); however, the amplitude of the $V$ profile of $6301.5 \AA$ is smaller than that of $6302.5 \AA$ in this case. A possible explanation is that the magnetic field is, on average, stronger in LB2 than in the broad part of LB1, approaching the Zeeman saturation limit for both lines. The amplitudes of the $V$ profiles would then be similar to each other. The slight difference between them is probably caused by different Landé factors, different temperatures in the line-formation regions, and by departures from LTE.

The stratifications of the magnetic field strength, which were computed with a stray-light profile different from that of the quiet sun, are presented with the dashed line in Figs. 2 and 3. In the case of the broad part of LB1 (Fig. 2), it corresponds to a stray light mixed with $20 \%$ of the profile taken from close umbra and $80 \%$ of the profile from the quiet sun. Higher contamination with umbral stray light caused a rapid increase of the merit function $\chi^{2}$ and an improbable stratification of plasma 

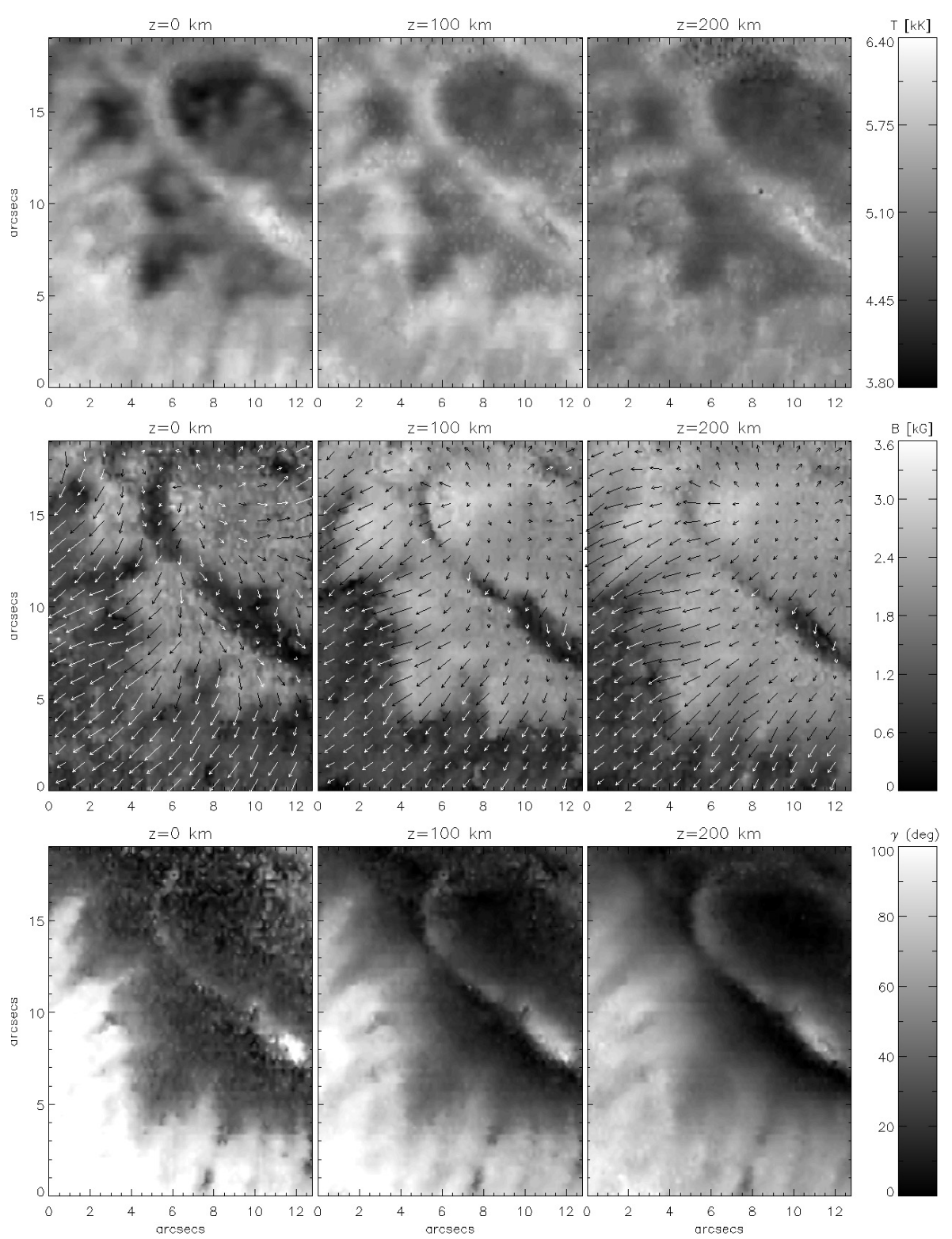

Fig. 4. The resulting maps of temperature (top), magnetic field strength with arrows indicating the projection of $\boldsymbol{B}$ to the solar surface (middle) and inclination (bottom) for area 1.

parameters. In the case of the narrow LB (Fig. 3), the stratification was obtained by using the stray-light profile only from the surrounding umbra, because there was no significant increase in the merit function in this case. We find that the increase in the fraction of the umbral component in the stray-light profile causes a decrease in magnetic field strength at almost all heights and a slight increase in the formation height of the canopy structure (see Sect. 4.2). But the main result, an increase in the field strength with height, remains unaltered. The increase in the umbral component in stray light also causes a logical increase in the temperature at all heights.

\subsection{Resulting maps of plasma parameters}

The spatial changes in temperature, magnetic field strength, and inclination are shown in Fig. 4 (area 1) and Fig. 5 (area 2). For the first time, the stratification with height for LBs is presented. We show the maps at three different heights in the atmosphere, where $0 \mathrm{~km}$ corresponds to $\log \tau=0$ (as set by SIR), $100 \mathrm{~km}$ corresponds to $\log \tau=-0.65$, and $200 \mathrm{~km}$ to $\log \tau=-1.3$ in LBs, on average.

Previous results concerning the temperature in the LB region were presented in the article by Rüedi et al. (1995), who studied the change in temperature along one cut through LB structure and found a temperature around $6400 \mathrm{~K}$ in the LB centre and a difference between LB and surrounding umbra of around $1500 \mathrm{~K}$ (all these values referred to $\tau_{1.56 \mu \mathrm{m}}=1$ ). We provide values of temperature averaged over various parts of the studied LBs in Table 1, along with average values for temperature in the surrounding umbra. The darkest parts of the umbral cores (continuum intensities smaller than 0.4 ) are considered in this case.

The temperature values inferred by SIR are lower than those in IR, which forms deeper in the atmosphere. Near $\tau_{5000}=1$ $(z=0 \mathrm{~km})$ narrow LBs have temperatures near $5500 \mathrm{~K}$ and broad LBs around $6000 \mathrm{~K}$. The central part of broad LBs reaches photospheric temperatures at this height. Comparison with the umbral cores is difficult as the cores on each side of LBs are very different. In area 1 , the umbral cores are filled by a large 


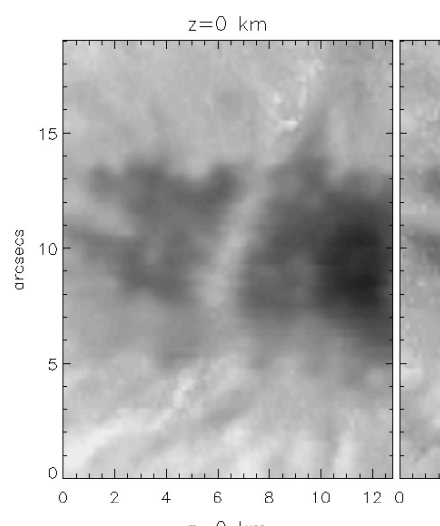

$z=100 \mathrm{~km}$
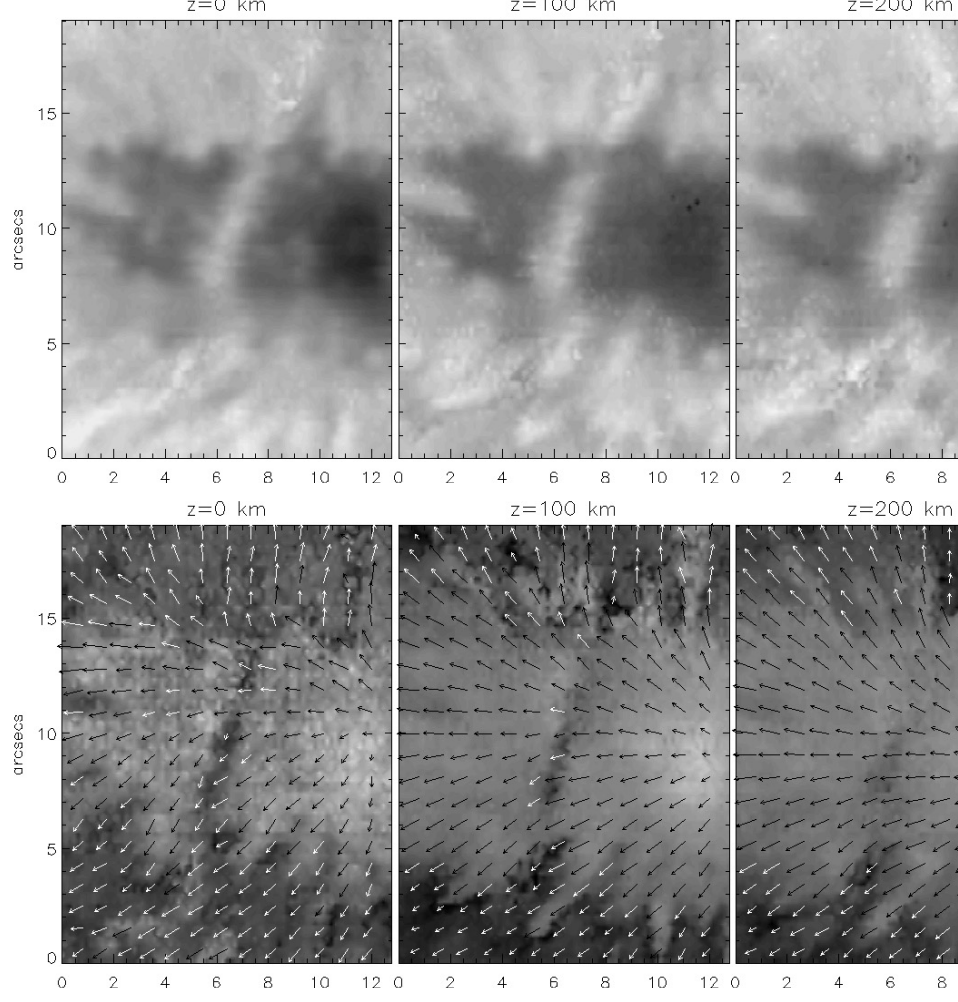
$z=100 \mathrm{~km}$
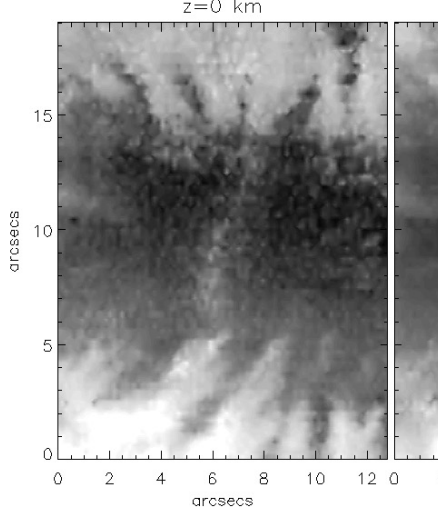

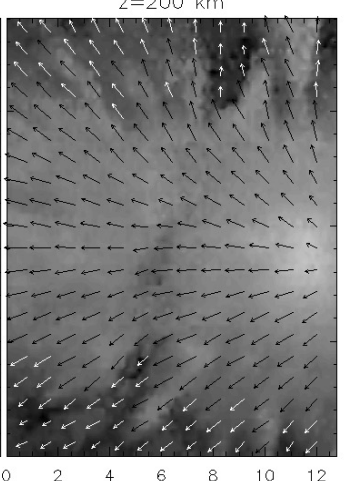

$z=200 \mathrm{~km}$
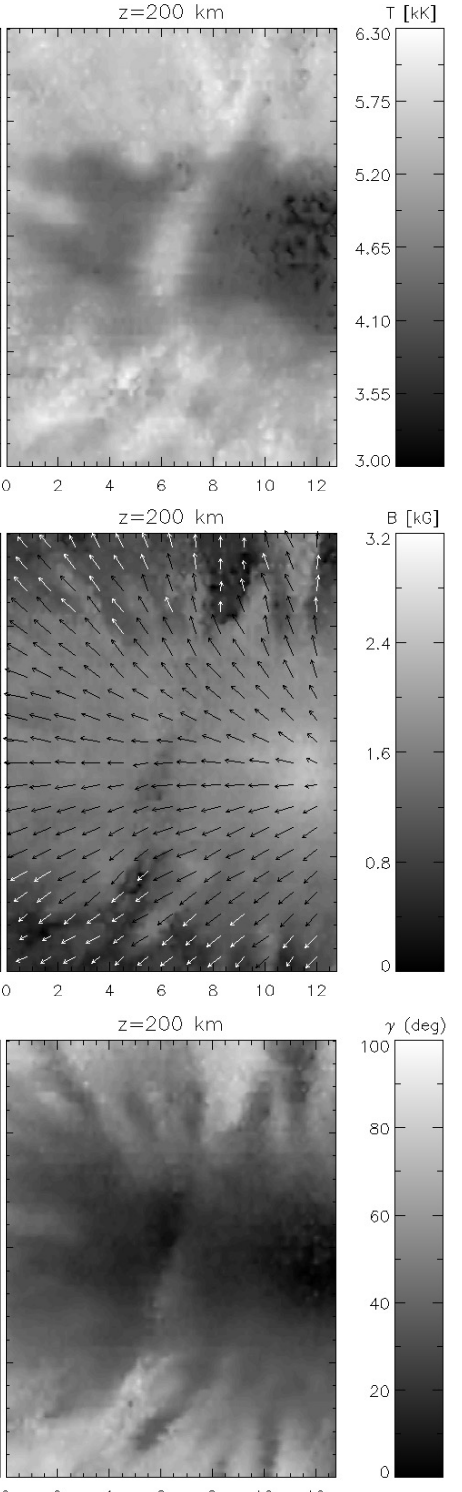

Fig. 5. Same as for Fig. 4 but for area 2.

number of dots (studied by Socas-Navarro et al. 2004). In area 2, one finds that umbral cores are almost free of umbral dots. The respective average temperatures are $4785 \mathrm{~K}$ and $4440 \mathrm{~K}$. In Table 1, additional temperatures at other heights are given.

The stratifications shown in Figs. 2 and 3 have a temperature bump at a height around $100-250 \mathrm{~km}$. The magnitude of this bump is typically $200 \mathrm{~K}$. The error bars retrieved by the inversion code are smaller than the amplitude of the bump, thus giving confidence to the existence of these bumps. Around $70 \%$ of the profiles inverted in the narrow parts of LBs show the bump at a height about of $120 \mathrm{~km}$ (similar to the one seen in Fig. 3). The situation for the broad part of LB1 shown in Fig. 2 is different. In this figure, the bump is higher, nearly $230 \mathrm{~km}$. While $32 \%$ of the points inside the bridge show this temperature bump, $37 \%$ of the points in broad LBs show a bump at a height of nearly $130 \mathrm{~km}$, as in the case of narrow LBs. Interestingly, these two different groups of points are spatially organised inside LB1, as can be seen in Fig. 4. Points with temperature bumps near $130 \mathrm{~km}$ are found near the border of LB, next to the umbral cores (top panel, $z=100 \mathrm{~km})$. At this height, a cold temperature island is seen in the middle of the bridge. The temperature inside the island is
$4850 \mathrm{~K}$, whereas in the bright rim it reaches $5400 \mathrm{~K}$. As we move to higher layers (same panel, $z=200 \mathrm{~km}$ ), the points with temperature bumps around this height are observed near the centre of broad LB1, closing down the island mentioned before. The bright regions at this height are as hot as before, near $5400 \mathrm{~K}$. The origin of such a structure is considered to be related to the canopy topology discussed in Sect. 4.2. Also, it is interesting to note that LBs are known to have brightenings in the chromosphere representing local heating sources (Berger \& Berdyugina 2003). The temperature bumps found here may be thought of as the upper photospheric counterpart to these temperature enhancements.

It is well known that LBs harbour magnetic fields with lower strengths and more inclined field lines. Beckers \& Schröter (1969) found a decrease in the magnetic field strength of $300 \mathrm{G}$ in a weak LB as compared to the surrounding umbra and an increase of inclination $\gamma \geq 5^{\circ}$. Rüedi et al. (1995) found a difference of $|B|$ between the umbra and LB around $1000 \mathrm{G}$ and a difference in inclination around $70^{\circ}$ in the LB centre. Leka (1997), using a Milne-Eddington inversion (which gives the results equivalent to the height of $\log \tau_{5000}=-1.5$ or 
Table 1. Summary of the average values of various plasma parameters in different regions of the studied LBs and surrounding umbrae.

\begin{tabular}{ccccccc}
\hline \hline Parameter & Height $[\mathrm{km}]$ & LB1 (narrow) & LB1 (broad) & Umbra 1 & LB2 & Umbra 2 \\
\hline$T$ & 0 & 5670 & 5990 & 4785 & 5490 & 4440 \\
{$[\mathrm{~K}]$} & 100 & 5250 & 5310 & 4280 & 5020 & 4000 \\
& 200 & 4890 & 5125 & 4100 & 4680 & 3740 \\
\hline$B$ & 0 & 1000 & 705 & 2200 & 1475 & 2640 \\
{$[\mathrm{Gauss}]$} & 100 & 1560 & 860 & 2240 & 1615 & 2600 \\
& 200 & 1700 & 1070 & 2150 & 1800 & 2620 \\
\hline$\gamma$ & 0 & 32 & 44 & 14 & 45 & 12 \\
{$[\mathrm{deg}]$} & 100 & 26 & 39 & 7 & 41 & 11 \\
& 200 & 25 & 35 & 8 & 41 & 16 \\
\hline$J_{z}$ & 100 & -94 & -17 & 14 & -41 & 3 \\
{$\left[\mathrm{~mA} \mathrm{~m}^{-2}\right]$} & 200 & -81 & -24 & 22 & -40 & 3 \\
\hline$\alpha_{\mathrm{rms}}$ & 100 & 0.6 & 1.7 & 0.4 & 0.7 & 0.2 \\
{$\left[\mathrm{Mm}^{-1}\right]$} & 200 & 0.4 & 1.0 & 0.2 & 0.4 & 0.2 \\
\hline
\end{tabular}

approximately $z=200 \mathrm{~km}$ ), found a range of decreases in $B$ from $300 \mathrm{G}$ to over $1200 \mathrm{G}$ and increased inclinations, usually by at least $10^{\circ}$.

The results presented in this paper show a similar behaviour but provide, for the first time, the stratification of the plasma parameters. The magnetic field is shown in Figs. 4 and 5 in the middle panel. One of the most striking features is how LBs (defined here as the regions with lower field strengths), in both figures, are seen to become narrower as we move to higher layers. At higher layers, LBs are less conspicuous as a field strength gap. Additionally, the field strength increases with height. In the centre of the broad LB, the field strength can be as low as $100 \mathrm{G}$ and increases to $700 \mathrm{G}$ at $z=200 \mathrm{~km}$. Averaged values are given in Table 1. All LBs show, almost everywhere, the same trend toward increasing field strength with height. Narrower bridges start from a higher average value at $z=0 \mathrm{~km}$ (1 kG in Fig. 3), but show the same trend.

As noted before, and in order to check if a field free $z=0 \mathrm{~km}$ atmosphere was also compatible for narrow LBs, we performed an inversion of the LB Stokes profiles where the stray light had various degrees of umbral core contributions (thus using straylight profiles including strong polarisation signals). Notably, the field strength near $z=0 \mathrm{~km}$ is reduced considerably, to levels smaller than $500 \mathrm{G}$ (the dashed line in the field strength stratification in Fig. 3). This shows that a completely field-free $z=0 \mathrm{~km}$ condition may not be fully discarded for even narrow ( $1^{\prime \prime}$ wide) LBs. The same procedure applied to broad LB shows zero field strength in the range $-20-50 \mathrm{~km}$ in the photosphere (see Fig. 2).

In agreement with previous authors, we also observed larger inclinations in all LBs. At the centre of broad LB1 at $z=100 \mathrm{~km}$, inclinations from $40^{\circ}$ to $90^{\circ}$ are found (bottom panel of Fig. 4). The tendency for the inclination is to decrease from this height upwards, ranging from $40^{\circ}$ to $70^{\circ}$ at $z=200 \mathrm{~km}$. Averaged inclination values are given in Table 1 . The same tendency is observed in narrow LBs albeit with more vertical fields. This tendency is not seen in Fig. 2, but it happens only at this pixel and a few others nearby. The broad LB region is mostly dominated by decreasing inclinations with height. As shown in Figs. 2 and 3 the inclination stratification is rather uncertain at $z=0 \mathrm{~km}$. The $1-\sigma$ errors are typically $\pm 20^{\circ}$, which makes it difficult to rely on them. Very often what we find is that the spline interpolation used by SIR forces the setting of a value by the trend of decreasing inclination with height. This is an artefact that originates the opposite polarity island seen in Fig. 4 in the inclination map at $z=0 \mathrm{~km}$, coordinates [12" $\left.7-8^{\prime \prime}\right]$. Note also that the field strength is quite low in these regions.
The azimuth of the field vector in LB follows the trend of the outward fanning field set by the dominating umbral core. In the broad part of LB1 there is a slight tendency for the azimuth to be more oriented toward the direction of LB, and to depart from the overall spread dictated by the umbral core. This tendency is seen at all heights, but more clearly near $z=0 \mathrm{~km}$, yet we note that this is the height with the largest uncertainties. It is interesting to mention that Leka (1997), who observed a larger number of LBs, found an alignment in several cases between the vector field azimuth and the direction set by LB.

\section{Discussion}

\subsection{Current density}

The $z$ component of the current density vector $\boldsymbol{J}$ can be computed from the inferred values of $B_{x}$ and $B_{y}$ :

$\mu J_{z}=(\nabla \times B)_{z}=\frac{\partial B_{y}}{\partial x}-\frac{\partial B_{x}}{\partial y}$,

where $\mu$ is the magnetic permeability. The resulting maps of $J_{z}$ are shown in Fig. 6. We present these maps only at the heights $z=100 \mathrm{~km}$ and $z=200 \mathrm{~km}$, because, as noted before, the errors in $\psi$ and $\gamma$ are too high at $z=0 \mathrm{~km}$. Because we infer the full atmosphere, one should be able to compute the full vector $\boldsymbol{J}$. But this step is non-trivial and will not be taken in this article, because both $J_{x}$ and $J_{y}$ involve partial derivatives with respect to the height $z$. But the $z$ scale is inferred by SIR assuming hydrostatic equilibrium stratification, which holds only along field lines. At photospheric heights, the plasma beta (the ratio between the gas and magnetic pressure) is not small and a force-free situation is hardly justifiable. Under such conditions the stratification will deviate from hydrostatic equilibrium making the $\mathrm{z}$ scale uncertain. A full derivation of the $\boldsymbol{J}$ vector must wait until a model with an assumed MHD configuration is constructed and the correct correspondence between optical depths and heights is made. Note also that such a model could also be used to properly account for the pixel to pixel fluctuations of the local Wilson depression, an effect not considered here.

Leka (1997) provided the first measurements of electric currents in LBs. The importance of these currents is that they provide a quantitative measurement of the disruption of the normal topology of the magnetic field in sunspots. She found typical values in a set of bridges ranging from 40 to $100 \mathrm{~mA} \mathrm{~m}^{-2}$. These currents are larger by as much as a factor of 10 than the normal values found in the umbra. Low-resolution magnetograms also find typical values not higher than a few times $10 \mathrm{~mA} \mathrm{~m}^{-2}$ 


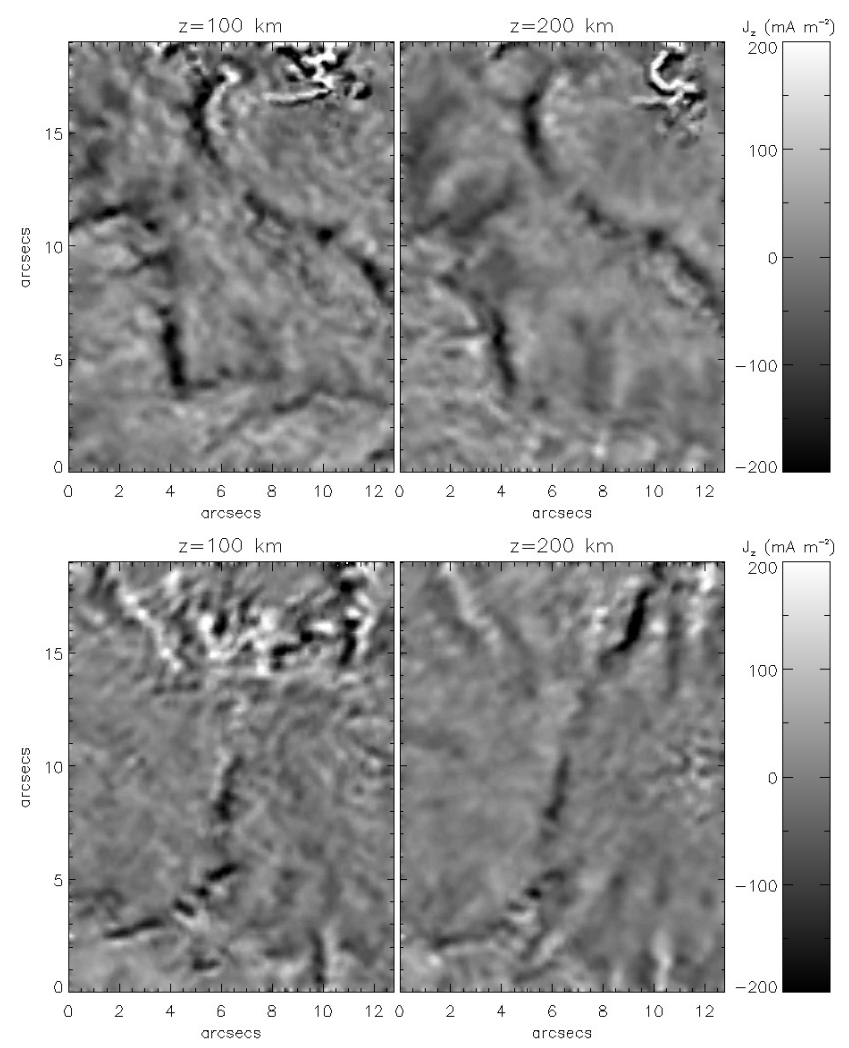

Fig. 6. Resulting maps of current density $J_{z}$ in area 1 (top) and area 2 (bottom).

(Georgoulis \& LaBonte 2004). In Table 1 we provide our mean values over selected regions inside LBs and nearby umbral cores. Typical errors in these estimates are in the range of $2-5 \mathrm{~mA} \mathrm{~m}^{-2}$. The LBs show mean values that are very much in the same range as those found by Leka (1997). For this positive polarity sunspot, they all show negative vertical currents dominating the LBs area. A few positive currents are seen. In contrast, the penumbral region shows a mixed positive and negative $J_{z}$ distribution. The two narrow bridges show mean currents that are much stronger than those in broad LB1. But current peaks are similar in all analysed LBs, reaching maximum values of $-200 \mathrm{~mA} \mathrm{~m}^{-2}$. These peaks are found more often at the $z=100 \mathrm{~km}$ than at the $z=200 \mathrm{~km}$ level (see the locations of strong currents in Fig. 6). The umbra shows mean currents that are smaller than $20 \mathrm{~mA} \mathrm{~m}^{-2}$, also in agreement with Leka (1997).

The existence of increased vertical currents (and most likely horizontal, too) suggests that Joule heating may be enhanced in these regions. Using our current densities, we computed mean values of Joule heating $J_{z} / \sigma$, where $\sigma$ is the electric conductivity (which in turn depends on other atmospheric parameters that are also retrieved by SIR). The estimate we obtained is about $10^{-3} \mathrm{erg} \mathrm{s}^{-1} \mathrm{~cm}^{-3}$. This energy should contribute to the radiative losses (i.e., the divergence of the radiative flux, Priest 1982 ) in the LB region. The magnitude of the radiative losses, which were calculated only from the $z$-component of radiative flux, was estimated to be $10^{4} \mathrm{erg} \mathrm{s}^{-1} \mathrm{~cm}^{-3}$. This is seven orders higher than the Joule heating contribution. Thus, at first sight one would not consider Joule heating as linked to the brightness of these structures, or, as found in this paper, to the temperature bumps in Sect. 3.2. However, two considerations must be taken into account in this regard. First of all, our resolution, hundreds of $\mathrm{km}$, is still inadequate for resolving relevant physical scales, as the presence of a current sheet would require. Second, we only estimate the vertical component of the electric currents. This may be the smallest of all the three components of the current vector. One might think that the two umbral cores on each side of LB are like close thick vertical flux tubes. These structures are known to have electric currents that lie basically in a plane perpendicular to the axis of the flux tube and distributed either throughout the whole volume or in a thin current sheet (Jahn 1997). Thus the vertical component that we see is most probably a small perturbation of the mostly horizontal current surrounding the flux tubes. In this case we would be heavily underestimating the amount of Joule heating. A proper account of this aspect can only be made using an MHD model of LB that estimates the total electric current from the jumps in the various magnetic parameters found in this work (along the lines of Hamedivafa 2003; Hamedivafa \& Sobotka 2004).

Related to the distribution of electric currents, we also computed mean values of the so-called force-free parameter $\alpha=$ $J_{z} / B_{z}$. As stressed by Leka (1997), it is this parameter that provides the input on the sign of the twist of the magnetic field in the LB areas. Normally, this parameter attains mean values for sunspots of the order of $0.05 \mathrm{Mm}^{-1}$, while $\delta$-spots reach much higher values. The distribution of $\alpha$ in our observations is similar to the $J_{z}$ distribution provided in Fig. 6 and is not shown here. In Table 1 we give rms values in LBs and umbral core regions. In LBs the vertical magnetic field is weak, and dividing by this magnitude to compute $\alpha$ amplifies the noise already found in the $J_{z}$ maps. It is for this reason that we provide the rms fluctuations of $\alpha$ here and not the mean values. The entries for the umbral cores given in this table should be used as the reference of the noise level for LB values. As can be seen, we find $\alpha$ to fluctuate at all heights more significantly in the LB regions than in the umbra. Broad LB has (at $z=100 \mathrm{~km}$ ) an rms of $\alpha$ around $1.7 \mathrm{Mm}^{-1}$ with peak-to-peak values in the range of $[-5,5] \mathrm{Mm}^{-1}$. These fluctuations become smaller with height, indicating a tendency for the magnetic field to become more potential.

\subsection{Canopy structure}

A magnetic canopy above LBs has already been suggested by Leka (1997), although her model of the atmosphere gives no stratification of plasma parameters with height. The basic idea behind introducing the canopy structure above LB is that if the field-free (or weak-field) plasma intrudes into the sunspot umbra, the umbral magnetic field fans out over such a region until it is blocked by the magnetic field of the opposite part of the umbra and forced again in the vertical direction. In Fig. 7 we illustrate such a canopy structure.

We see in Figs. 4 and 5 (middle panel) that the magnetic field strength is weak in the lowest layers in all parts of LBs. The area filled with the weak-field plasma is contracting with height. This means that the broad part of LB1 reaches at $z=200 \mathrm{~km}$ a similar width to the narrow part of this $\mathrm{LB}$ at $z=0 \mathrm{~km}$. The narrowest parts of LBs are almost indistinguishable at $z=200 \mathrm{~km}$. The temperature enhancements found by the inversions occupy a smaller area as one moves to higher layers. The inclination maps (Figs. 4 and 5, bottom panel) give a similar visual impression to the magnetic field strength maps. Generally, the inclination decreases with height in all parts of LBs, and in the narrow parts it acquires values that are similar to those in the surrounding umbra. The arrows in Figs. 4 and 5 (middle panel) indicate the magnetic field azimuth. The case represented in Fig. 7 is a simplistic, $2 \mathrm{D}$ scenario that becomes more complex in real sunspots. In particular, the field lines do not necessarily have to meet as nicely as depicted in our 2D picture. This is seen in the LBs studied 


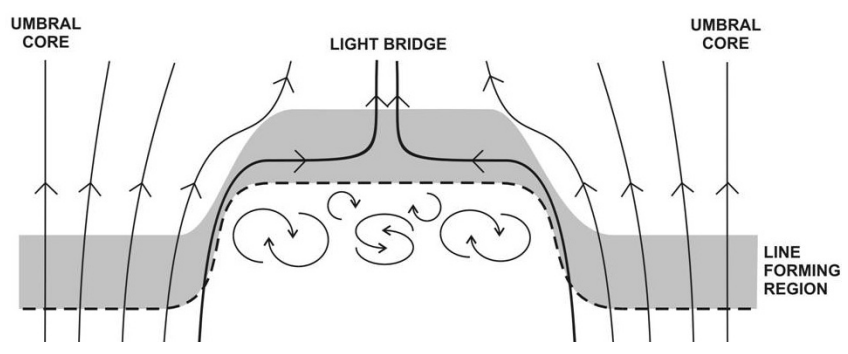

Fig. 7. Schematic view of the magnetic canopy above LB.

here. In both cases, we observe LB surrounded by well-defined umbral cores. The azimuth directions in LB are dominated by the spread dictated by these umbral cores. The distortion introduced in the field azimuth by LBs is seen to be small, and almost negligible for narrow structures. Only the broad part of LB1 is recognisable in azimuth maps and shows the magnetic field that is more aligned with the LB axis.

The presence of this canopy structure has to create disruptions in the magnetic field and therefore higher values of $J_{z}$. The highest values of current densities can be expected at the border between the magnetic field of the umbra and the field-free plasma in LB. This is what one can see in Fig. 6, where the maximum currents do not coincide with the central part of the broad LB1, but with its boundaries. The currents are negligible at the centre of LB at this spatial resolution.

The facts presented in the previous paragraphs are in good agreement with the diagram in Fig. 7. We can assume that the only difference between the narrow and broad parts of LBs is the height of emerging field-free plasma. In the narrow parts of LBs, the field-free plasma reaches low layers of the line-formation region. In the case of broad LBs, it penetrates through the lineformation region, and the width of field-free plasma region decreases with height. It is important to note here that by using umbral stray light we found that the magnetic field in the lowest layers of narrow LBs is also compatible with the zero field.

\section{Conclusions}

Two strong LBs in a complex irregular sunspot were observed spectropolarimetrically with a high spatial resolution of 0 .' 7 . The full Stokes profiles were inverted, yielding three-dimensional information about the temperature and magnetic field vector in LBs and their surroundings. The resulting magnetic field strength and orientation coincide with the expected behaviour in the umbra and penumbra. The changes in magnetic field strength and inclination in LBs agree well with previous studies of these phenomena. All this lends confidence to the results from the inversion code. Another demonstration of one-dimensional inversion code validity is the smoothness of the maps of the temperature and magnetic field (strength, inclination and azimuth).

We find local enhancements in the temperature stratification (bumps) in about $60 \%$ of the points inside LBs. This can be seen in Fig. 2 around $z=200 \mathrm{~km}$ and in Fig. 3 around $z=100 \mathrm{~km}$. Taking into account the very small uncertainties in temperature calculated by the inversion code, we believe that these enhancements are not artifacts of the inversion method but real phenomena. It is interesting how strongly the magnetic field changes in LBs even over the small height range of $200 \mathrm{~km}$. The magnetic field strength in the umbra decreases with height $\left(-0.8 \mathrm{G} \mathrm{km}^{-1}\right)$, while in LBs it rapidly increases with gradients reaching $4.7 \mathrm{G} \mathrm{km}^{-1}$. The field is very weak in LBs at $z=0 \mathrm{~km}$ but is nearly equal to that of the umbra at $z=200 \mathrm{~km}$. Also the inclination shows considerable changes with height. The magnetic field is very inclined at low levels (especially in broad LB1), but at high levels it has a similar inclination to the one in the surrounding umbra (especially for narrow LBs). The resulting stratification of a magnetic field vector with height indicates the presence of a magnetic canopy above the field-free or weakfield plasma that forms both LBs. We stress that this work has indeed found, for the first time, field-free regions in the broad part of LB1 and evidence of similar conditions in narrower LBs.

These LBs are regions with enhanced vertical electric currents (and force-free parameter $\alpha$ ). We have derived the highest currents found in non- $\delta$ spots (up to $200 \mathrm{~mA} \mathrm{~m}^{-2}$ ). This is most probably due to the relatively high spatial resolution achieved by our observations. But even these currents cannot account (through Joule heating) for the temperature bumps found by the inversion code. As pointed out before, better resolution and an estimate of the full $\boldsymbol{J}$ vector is fundamental to resolving this issue. This is important in the context of the ubiquitous brightness enhancements observed in the chromosphere by TRACE (Berger $\&$ Berdyugina 2003). These authors found that LBs have chromospheres that are magnetically heated as they are constantly pervaded by brightenings in the $1600 \AA$ images. This band forms in a temperature range from $4000 \mathrm{~K}$ to $20000 \mathrm{~K}$, that is, from the upper photosphere to well into the chromosphere. Thus our temperature bumps may be related to these chromospheric brightenings. Their physical origin can be linked to the canopy topology also found in this work. As explained above, this topology can be very complex in $3 \mathrm{D}$, harbouring field lines that meet at large angles, where reconnection can occur. It is also interesting to note that convective motions in LBs are very dynamic, so one can see the boundaries between the umbral cores and LBs in constant change (Berger \& Berdyugina 2003). Accordingly, the canopy structure above is subject to constant readjustment, which in turn can form the required heating source. From an observational point of view, the combination of uninterrupted chromospheric TRACE observations and high-spatial resolution spectropolarimetric data from the Japanese-led solar-B mission (launch 2006) represents our best opportunity to gain further insight into this problem.

Acknowledgements. The authors thank Basilio Ruiz Cobo for advice on the Joule heating and net radiation derivations along with the useful guidance on the SIR code. This work was supported by the research project IAA3003404 of the Grant Agency of the the Academy of Sciences of the Czech Republic and by the EARA Early Stage Training program. This research is a part of the European Solar Magnetism Network (EC contract HPRN-CT-2002-00313) and part of the research project AV0Z10030501 of the Astronomical Institute of the Academy of Sciences of the Czech Republic. This work is partly funded by the Spanish ESP2003-07735. The Swedish Vacuum Solar Telescope was operated on the island of La Palma by the Institute for Solar Physics of the Royal Swedish Academy of Sciences in the Spanish Observatorio del Roque de los Muchachos of the Instituto de Astrofísica de Canarias.

\section{References}

Beckers, J. M., \& Schröter, E. H. 1969, Sol. Phys., 10, 384 Bellot Rubio, L. R. 1999, in A user quide to SIR Berger, T. E., \& Berdyugina, S. V. 2003, ApJ, 589, L117 Bray, R. J., \& Loughhead, R. E. 1964, Sunspots, The International Astrophysics Series (London: Chapman \& Hall)

Bumba, V., \& Hejna, L. 1980, Bull. Astron. Inst. Czech., 31, 257 
Bumba, V., \& Suda, J. 1983, Bull. Astron. Inst. Czech., 34, 29

García de La Rosa, J. I. 1987, Sol. Phys., 112, 49

Georgoulis, M. K., \& LaBonte, B. J. 2004, ApJ, 615, 1029

Hamedivafa, H. 2003, A\&A, 407, 761

Hamedivafa, H., \& Sobotka, M. 2004, A\&A, 428, 215

Hirzberger, J., Bonet, J. A., Sobotka, M., Vázquez, M., \& Hanslmeier, A. 2002, A\&A, 383, 275

Jahn, K. 1997, in 1st Advances in Solar Physics Euroconference, Advances in Physics of Sunspots, ASP Conf. Ser., 118, 122

Leka, K. D. 1997, ApJ, 484, 900

Lites, B. W., Bida, T. A., Johannesson, A., \& Scharmer, G. B. 1991, ApJ, 373, 683

Lites, B. W., Low, B. C., Martinez Pillet, V., et al. 1995, ApJ, 446, 877

Martínez Pillet, V., Collados, M., Sánchez Almeida, J., et al. 1999, in High Resolution Solar Physics: Theory, Observations, and Techniques, ASP Conf. Ser., 183, 264

Martínez Pillet, V., Trujillo Bueno, J., \& Collados, M. 2001, in Advanced Solar Polarimetry - Theory, Observation, and Instrumentation, ASP Conf. Ser., 236,133
Press, W. H., Flannery, B. P., \& Teukolsky, S. A. 1986, Numerical recipes, The art of scientific computing (Cambridge: University Press)

Priest, E. R. 1982, Solar magneto-hydrodynamics (Dordrecht, Holland; Boston, Hingham: D. Reidel Pub. Co.), 74P

Rimmele, T. R. 1997, ApJ, 490, 458

Rüedi, I., Solanki, S. K., \& Livingston, W. 1995, A\&A, 302, 543

Ruiz Cobo, B. 1998, Ap\&SS, 263, 331

Ruiz Cobo, B., \& del Toro Iniesta, J. C. 1992, ApJ, 398, 375

Scharmer, G. B., Pettersson, L., Brown, D. S., \& Rehn, J. 1985, Appl. Opt., 24, 2558

Sobotka, M., Bonet, J. A., \& Vazquez, M. 1993, ApJ, 415, 832

Sobotka, M., Bonet, J. A., \& Vazquez, M. 1994, ApJ, 426, 404

Socas-Navarro, H., Pillet, V. M., Sobotka, M., \& Vázquez, M. 2004, ApJ, 614, 448

Westendorp Plaza, C., del Toro Iniesta, J. C., Ruiz Cobo, B., et al. 1998, ApJ, 494, 453

Westendorp Plaza, C., del Toro Iniesta, J. C., Ruiz Cobo, B., et al. 2001, ApJ, 547,1130

Zirin, H., \& Wang, H. 1990, Sol. Phys., 125, 45 